\title{
Hydrogen Induced Degradation: A Possible Mechanism for Light- and Elevated Temperature-induced Degradation in n- type Silicon
}

Daniel Chen $^{* a}$, Phillip G. Hamer ${ }^{\mathrm{b}}$, Moonyong Kimª ${ }^{\mathrm{a}}$ Tsun H. Fung ${ }^{\mathrm{a}}$, Gabrielle Bourret-Sicotte ${ }^{\mathrm{b}}$, Shaoyang Liu ${ }^{\mathrm{a}}$, Catherine E. Chan ${ }^{\mathrm{a}}$, Alison Ciesla ${ }^{\mathrm{a}}$, Ran Chen ${ }^{\mathrm{a}}$, Malcolm D. Abbott ${ }^{\mathrm{a}}$, Brett J. Hallam ${ }^{\mathrm{a}}$ and Stuart R. Wenham ${ }^{\mathrm{a}}$

aSchool of Photovoltaic and Renewable Energy Engineering, The University of New South Wales, Kensington, NSW, 2052, Australia

bepartment of materials, University of Oxford, Parks Rd, Oxford OX1 4NH, United Kingdom

*Corresponding author e-mail: daniel.chen@unsw.edu.au Phone: +61 450088 104, Fax: +61 293857762

\begin{abstract}
In this work, we demonstrate a form of minority carrier degradation on ntype $\mathrm{Cz}$ silicon that affects both the bulk and surface related lifetimes. We identify three key behaviors of the degradation mechanism; 1) a firing dependence of degradation extent, 2) the appearance of bulk degradation when wafers are fired in the presence of a diffused emitter and 3) a firing related apparent surface degradation when wafers are fired in the absence of an emitter. We further report a defect capture cross-section ratio of $\sigma_{n} / \sigma_{p}=0.028 \pm 0.003$ for the defect in n-type. Utilizing our understanding of LeTID in p-type silicon, we demonstrate that the degradation behaviors in both n-type and p-type silicon are closely correlated. In light of numerous reports on the involvement of hydrogen the potential role of a hydrogen-induced degradation mechanism is discussed in both p- and n-type silicon, particularly in relation to the diffusion of hydrogen and influence of hydrogen-dopant interactions.
\end{abstract}

Keywords: LeTID; n-type silicon; hydrogen; emitter diffusion; hydrogen-induced degradation.

\section{Introduction}

Light- and elevated temperature-induced degradation (LeTID) has generated significant interest in both academic and industrial circles of the photovoltaics industry. Initially, this phenomenon was observed to negatively impact the lifetime and electronic characteristics of devices and wafers fabricated on p-type multi-crystalline silicon wafers, especially on those utilizing the higher efficiency passivated emitter and rear contact (PERC) cell technology [1]. Numerous studies have undertaken detailed defect analysis studies and defect formation and recovery kinetics in an effort to identify a possible root cause [2-6]. A key behavior is the modulation of the extent of LeTID with firing temperature, with an increasing extent of degradation with increasing firing temperature, and the absence of degradation in unfired samples [2,3]. Many recent studies have also postulated the involvement of hydrogen in the formation of and as a possible root cause for LeTID, although the true nature of the involvement of hydrogen is far from understood [7-10]. Kersten et al. demonstrated that LeTID was only observed on samples that had been fired in the presence of a hydrogen containing dielectric layer such as passivating hydrogenated silicon nitride $\left(\mathrm{SiN}_{\mathrm{x}}: \mathrm{H}\right)$ and aluminium oxide $\left(\mathrm{AlO}_{\mathrm{x}}: \mathrm{H}\right)$ films [11]. Vargas et al. demonstrated a manipulation of the LeTID related degradation by varying the hydrogen fraction in $\mathrm{SiN}_{\mathrm{x}}$ : $\mathrm{H}$ films, thus showing a correlation between degradation extent and the amount of hydrogen released from the dielectric [12]. Hydrogen as a component of the LeTID defect would be in agreement with 
the strong firing dependency of LeTID; a greater fraction of hydrogen bonds in the dielectric layers are broken at higher temperatures beyond a threshold temperature of approximately $600{ }^{\circ} \mathrm{C}$ where hydrogen release from the dielectric is known to start [13].

Of particular interest for this work are the recent observations of Bredemeier et al. [5]. Their results provided evidence that the recovery in lifetime after degradation was driven by the diffusion of some species to the wafer surface. They highlighted that nickel or cobalt could possibly be the fast diffusing defect, although they could not rule out the involvement of hydrogen. However, it was suggested that, instead, the in-diffusion of hydrogen from the surface would result in the passivation of the defect.

Recent studies by Chen et al. and Fertig et al. have shown that the identical defect can also manifest itself within solar cells fabricated on p-type Czochralski-grown silicon [14,15]. Since then, similar observations of degradation on float-zoned (FZ) silicon have been proposed to arise from identical defects [16-18]. In these FZ-LID studies, Sperber et al. observed an initial bulk deterioration followed by subsequent declines in surface passivation quality [18].

N-type silicon has been long lauded for its low susceptibility to light-induced degradation such as boron-oxygen related degradation and the impact from metallic contaminants [19]. Although this material does indeed suffer from degradation induced by grown-in defects, which have been extensively studied [20], an investigation of LeTID on n-type silicon concluded that the defect either does not manifest itself or is not recombination active in ntype [17]. On the other hand, a study by Renevier et al. demonstrated a carrier-induced instability in the lifetime of boron-diffused n-type wafers fired in the presence of a hydrogenrich dielectric when subject to either illuminated annealing or dark annealing [21]. Although it was determined that phosphorus diffusions resulted in a suppression of the degradation phenomenon, and that degradation did not occur in the presence of a silicon-rich silicon (lowhydrogen) nitride or aluminium oxide layer, a lack of understanding of the defect and of LeTID at the time resulted in unclear conclusions.

In this paper, we further extend the work of Renevier et al. [21]. With increased understanding of LeTID and its behavior under various testing conditions, we demonstrate that n-type silicon can be susceptible to LeTID, with behaviors akin to the defect observed in studies on p-type silicon. We investigate the importance of a diffused emitter both during firing and LeTID testing and correlate observed behaviors with various models described in literature. In light of recent work suggesting the involvement of hydrogen as a cause of LeTID, we explain the possible diffusion mechanisms behind a highly plausible hydrogen induced degradation.

\section{Methodology}

\subsection{Sample Preparation}

To carry out our investigations of LeTID, symmetrical lifetime precursors were fabricated on commercially acquired silicon wafers. For n-type specimens, we used neighboring $156 \mathrm{~mm} \times$ $156 \mathrm{~mm}$ uncompensated n-type phosphorus-doped $2 \Omega . \mathrm{cm}$ Czochralski-grown wafers with a thickness of $190 \pm 2 \mu \mathrm{m}$ obtained from Woongjin Energy. These as-cut wafers were placed in 
potassium hydroxide solution to remove saw damage and subsequently textured to a final thickness of $183 \pm 3 \mu \mathrm{m}$ in an alkaline texturing bath. The wafers were then cleaned using the radio corporation of America (RCA) 1 and 2 processes, that is; a 6 min dip in ammonium hydroxide $\left(\mathrm{NH}_{3} \mathrm{OH}\right)$ and hydrogen peroxide $\left(\mathrm{H}_{2} \mathrm{O}_{2}\right)$ solution and a subsequent 6 min clean in a solution of hydrochloric acid $(\mathrm{HCl})$ and hydrogen peroxide. This is then followed by a short HF dip to remove any native oxides. The wafers were then split into 3 groups. Wafers in Group 1 received a boron diffusion in a boron tribromide $\left(\mathrm{BBr}_{3}\right)$ quartz-tube furnace at temperatures of $\approx 920{ }^{\circ} \mathrm{C}$ resulting in a $\mathrm{p}^{+}$-emitter with a sheet resistance of $\mathrm{R}_{\text {sheet }}=(95 \pm 5)$ $\Omega /$ sq. Using these conditions, we avoid generating SRH recombination centers from misfit dislocations described by Cousins and Cotter [22]. Afterwards, dilute hydrofluoric acid (DHF) (2\% v/v) was used to remove borosilicate glass from the surface. Group 2 wafers were also diffused in a phosphoryl chloride $\left(\mathrm{POCl}_{3}\right)$ diffusion tube to apply a phosphorus diffused $\mathrm{n}^{+}$-emitter with sheet resistance of $\mathrm{R}_{\text {sheet }}=(60 \pm 3) \Omega /$ sq. Similarly, DHF was used to remove any phosphosilicate glass from the surface. Group 3 wafers did not receive any diffusion process. All wafers were then RCA cleaned again before deposition of dielectric layers. A 75 $\mathrm{nm}$ layer of hydrogenated silicon nitride $\left(\mathrm{SiN}_{\mathrm{X}}: \mathrm{H}\right)$ film with a refractive index of 2.08 at 633 nm [23] was deposited on both sides using a Meyer Burger MAiA remote plasma enhanced chemical vapor deposition (r-PECVD) tool [24]. Once deposited, wafers were fired in an inline Schmid metallization fast-firing belt furnace at a range of setpoint temperatures between $700{ }^{\circ} \mathrm{C}$ and $900{ }^{\circ} \mathrm{C}$ (exact temperatures between $640{ }^{\circ} \mathrm{C}$ and $835{ }^{\circ} \mathrm{C}$ ) and a set conveyer speed of $4.5 \mathrm{~m} / \mathrm{min}$. Actual temperatures were measured on identical dummy wafers using a Q13 Datapaq temperature profiler averaged using three K-type thermocouples (Omega KMQXLIM075G-300). Additional unfired wafers were kept as controls. The six-inch wafers were then laser cleaved into $52 \mathrm{~mm} \times 52 \mathrm{~mm}$ tokens. The central token from each substrate was taken for experimental use due to variations in sheet resistivity from diffusion nonuniformities around the edge of the 6-inch wafer. As a comparison to n-type, a set of p-type high-performance multi-crystalline wafer acquired from GCL Energy Holdings in the same group to those used in [15] were processed in parallel. These wafers were $156 \mathrm{~mm} \times 156 \mathrm{~mm}$ boron-doped wafers with a base resistivity of $1.6 \pm 0.1 \Omega$.cm and thickness of $192 \pm 4 \mu \mathrm{m}$. Adjacent 'sister' wafers were chosen from the ingot to ensure similar crystallographic and electrical properties. After texturing in acidic solution, these multi-crystalline silicon wafers were processed in an identical fashion to those in Group 2 described above.

\subsection{Testing for LeTID}

Initial testing for LeTID behaviour was carried out using a dark annealing process proposed by [9] and [15]. Wafers were placed on a hotplate in the dark and annealed at a temperature of $175 \pm 5{ }^{\circ} \mathrm{C}$. In parallel, a conventional light soaking process at elevated temperature was used to test for LeTID under illumination. Samples were exposed to light with an illumination intensity of $0.96 \mathrm{~kW} / \mathrm{m}^{2}$ (S305C, Thorlabs) supplied using four broadband halogen lamps whilst on a hotplate heated to approximately $75 \pm 2{ }^{\circ} \mathrm{C}$. Ex-situ quasi-steadystate photoconductance (QSSPC) measurements were carried out periodically during LeTID testing using a photoconductance tool (Sinton Instruments, WCT-120TS) [25]. Measurements of minority carrier lifetime were analysed using the generalised method proposed by [26] and 
references therein and intrinsic recombination was corrected using the model developed by Richter et al. [27]. Effective minority carrier lifetimes $\left(\tau_{\text {eff }}\right)$ were extracted at an injection level $(\Delta n)$ equal to approximately 0.1 times the background dopant density $\mathrm{N}_{\mathrm{d}}$ (i.e. $\Delta n=2.4 \times$ $10^{14} / \mathrm{cm}^{3}$ for n-type and $\Delta n=9.1 \times 10^{14} / \mathrm{cm}^{3}$ for p-type samples). The apparent normalized defect density (NDD) metric was used to evaluate the evolutions in effective carrier lifetime between samples [28]:

$$
\operatorname{NDD}(\Delta n)=\sigma_{\mathrm{n} / \mathrm{p}} v_{\mathrm{th}} N_{\mathrm{t}}=\frac{1}{\tau_{\text {eff }}(\Delta n)}-\frac{1}{\tau_{\text {fired }}(\Delta n)}
$$

where $N_{\mathrm{t}}$ is the defect concentration, $v_{\mathrm{th}}$ is the thermal velocity of carriers and $\sigma_{\mathrm{n} / \mathrm{p}}$ is the capture cross section of the defect. For our investigation, we will denote $\tau_{\text {eff }}$ as the effective minority carrier lifetime measured during LeTID testing and $\tau_{\text {fired }}$ as the lifetime measured directly after firing and before any additional thermal processes. Note that this assumption will assume that the amount of LeTID that may be formed during the firing process [15] is negligible compared to the quantity formed during testing. The same photoconductance tool was also used to carry out in-situ monitoring of dark annealing processes at elevated temperatures. Due to the temperature dependence of carrier mobility, a correction based upon the Dorkel-Leturcq mobility model [29] was applied to measurements taken at elevated temperatures $\left(>30^{\circ} \mathrm{C}\right)$ to ensure optimal calibration of the instrument.

\section{Results}

\subsection{Impact of emitter diffusion on LeTID in n-type}

The aim of the initial investigation was to observe degradation in n-type when wafers were subjected to different diffusion treatments and under various testing conditions. Fig. 1 (a) shows the evolution in carrier lifetime for samples treated in the dark at elevated temperatures. No degradation is observed for samples with a phosphorus diffusion. Samples with boron diffusions, however, exhibit a strong bulk degradation and recovery mechanism throughout the annealing process. A maximum defect concentration is reached after approximately $10 \mathrm{~h}$ followed by a complete recovery of the sample lifetime after $280 \mathrm{~h}$. No significant changes of the surface dark saturation current density $\left(\mathrm{J}_{0 \mathrm{~s}}\right)$ were observed, suggesting that the degradation occurred within the bulk. The non-diffused wafers also exhibited a gradual degradation in effective lifetime, however this was found to be dominated by a degradation in the surface with a $J_{0 s}$ increase from $114 \mathrm{fA} / \mathrm{cm}^{2}$ to $133 \mathrm{fA} / \mathrm{cm}^{2}$ and with a stable bulk lifetime of $330 \pm 5 \mu$ s. The probable cause for the observed degradation will be discussed later. Phosphorus diffused samples appear to be stable with extended dark anneal treatments, showing no signatures of degradation at the surface or in the bulk.

Under illumination, similar behaviors were observed on the boron-diffused and non-diffused samples as seen in Fig.1 (b). A maximum degradation on boron-diffused samples was reached after approximately $20 \mathrm{~h}$, which was then followed by a period of lifetime recovery. This degradation time scale, however, appears to be significantly faster than what others have reported for the degradation in p-type silicon under the same conditions (Chan et al. reported close to $300 \mathrm{~h}$ for p-type multicrystalline silicon) [3]. The non-diffused samples did not 
display the typical degradation and recovery associated with LeTID, which is consistent with results in other works that did not observe a manifestation on LeTID in n-type [16]. However, the non-diffused samples again suffer from a decrease in surface passivation quality. In contrast, the n-type phosphorus-diffused samples display a rapid degradation, reaching its minimum with a time scale of approximately $11 \mathrm{~h}$, followed by a recovery to its initial performance within $180 \mathrm{~h}$. From this observation, we note the possibility that dark annealing at $\sim 172{ }^{\circ} \mathrm{C}$ may have accelerated the degradation and regeneration rates beyond what may be observable within our time scales. A further improvement in $\tau_{\text {eff }}$ after light soaking beyond $180 \mathrm{~h}$ was also observed, which may be attributed to the removal of defects pre-formed during the firing process or to the hydrogen passivation of other bulk impurities [15]. Nonetheless, the results show consistency with what was observed by Renevier et al. [21].
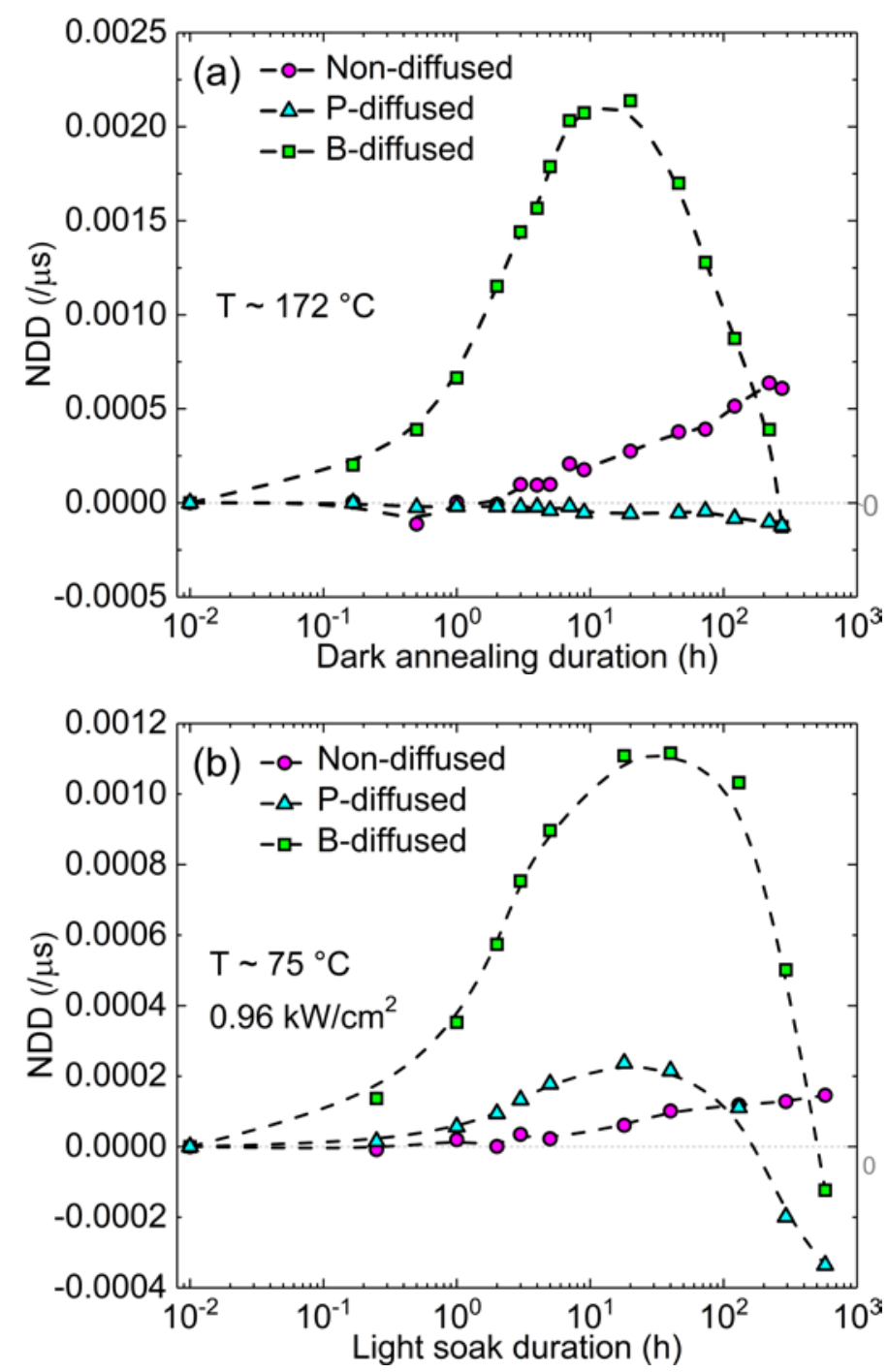

Fig. 1 (a) Normalised defect density of n-type silicon lifetime structures with a boron diffusion (square), a phosphorus diffusion (triangle) or no diffusion (circle) vs. dark annealing duration at 172 ${ }^{\circ} \mathrm{C}$ after firing at $744{ }^{\circ} \mathrm{C}$ and (b) the same substrate types exposed to an illuminated annealing process of $75{ }^{\circ} \mathrm{C}$ and illumination intensity $0.96 \mathrm{~kW} / \mathrm{cm}^{2}$. Effective carrier lifetimes were extracted at an injection level of $\Delta n=2.4 \times 10^{14} / \mathrm{cm}^{3}$. Lines are shown to serve as a guide to the eye. 


\subsection{Firing dependence of degradation and comparisons to p-type silicon}

An investigation on the possible firing dependence was carried out on boron-diffused n-type wafers to identify whether the observed degradation held similar characteristics to the LeTID reported in p-type silicon. The change in normalized effective lifetime with dark annealing time is shown in Fig. 2. The boron-diffused n-type wafers show a distinct correlation with firing condition with the extent of degradation being more profound at higher temperatures (Fig. 2 (a)).
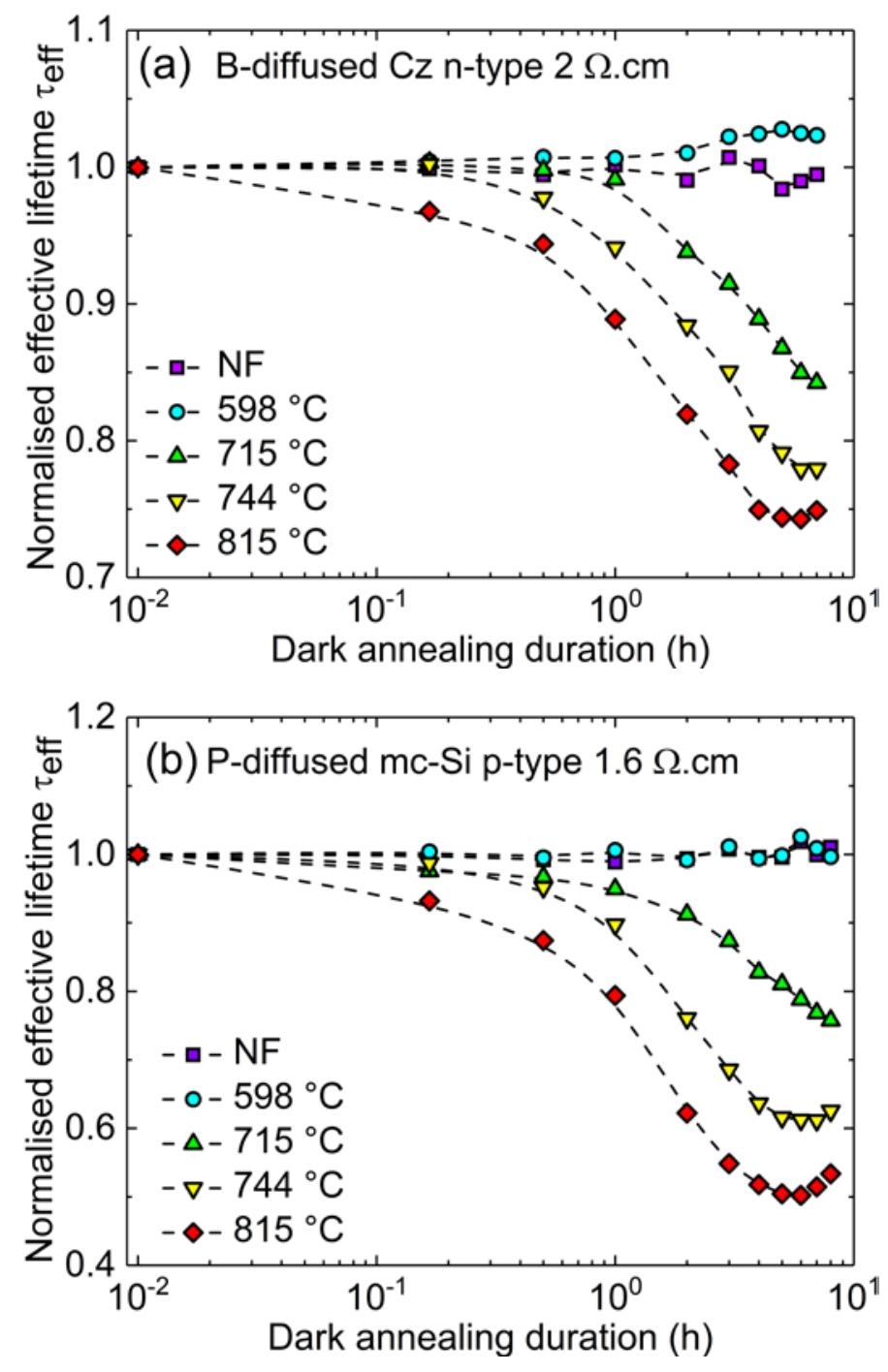

Fig. 2 (a) Normalised effective minority carrier lifetimes vs. dark annealing duration at $175{ }^{\circ} \mathrm{C}$ for (a) boron-diffused n-type $\mathrm{Cz}$ wafers and (b) phosphorus-diffused p-type multicrystalline-silicon wafers that have been fired at various temperatures (from $598{ }^{\circ} \mathrm{C}$ to $815{ }^{\circ} \mathrm{C}$ ) or left unfired as control. Temperatures in the legend are actual measured substrate temperatures. Lines are shown to serve as a guide to the eye.

In strong agreement with other LeTID studies on p-type multi-crystalline silicon, the degradation is also suppressed or no longer prevalent when unfired or when firing temperatures are lowered below $700{ }^{\circ} \mathrm{C}$ [2,3]. In comparison to the defect formation observed on p-type multi-crystalline silicon (see Fig. 2 (b)), the time scales required to reach a lifetime minimum is also well matched between the two materials. This further validates the 
possibility that identical defects causing LeTID in p-type are also responsible for the degradation in n-type. The injection-dependent lifetime spectroscopy (IDLS) analysis of a degraded boron-diffused sample is depicted in Fig. 3. The injection-dependent inverse SRH lifetime $\left(1 / \tau_{\mathrm{SRH}}\right)$ was realized through a linear subtraction of the inverse carrier lifetimes measured after firing $\left(1 / \tau_{\text {meas_init }}\right)$ and after degradation via dark annealing $\left(1 / \tau_{\text {meas_DA }}\right)$. By using the assumption of a deep-level defect situated near mid-gap, we report a defect with an asymmetric electron-to-hole capture cross-section ratio of $k=\sigma_{\mathrm{n}} / \sigma_{\mathrm{p}}=0.028 \pm 0.003$ or an equivalent hole-to-electron ratio of $k=35 \pm 4$. Surprisingly, this value is quite similar to the $Q$-factors identified for the defect responsible for LeTID in p-type multi-crystalline silicon and FZ silicon in various other works $[8,15,16,30]$. Given the strong asymmetric behavior of the capture cross-section ratio in p-type silicon, one would not expect such strong injectiondependence behavior to be obtained in n-type silicon with accordance to Shockley-Read Hall theory. However, this finding does not detract from the possibility that the defect may occupy multiple energy levels (such as a "negative-U” type impurity) and further analysis of the recombination parameters beyond the scope of this paper will be required.

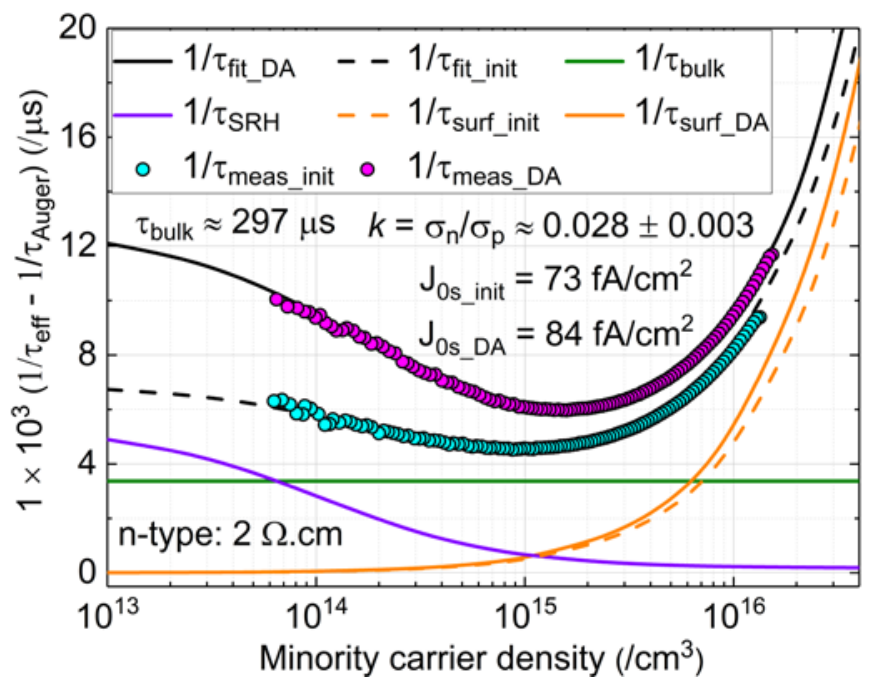

Fig. 3 Auger-corrected injection-dependent lifetime spectroscopy of a boron-diffused n-type substrate fired at a temperature of $815^{\circ} \mathrm{C}$ measured prior to degradation $\left(1 / \tau_{\text {meas_init }}\right)$ and degraded using $175^{\circ} \mathrm{C}$ DA to the minimum lifetime $\left(1 / \tau_{\text {meas_DA }}\right)$. A fit of the measured data is denoted by the black solid and dashed line whereas the other curves denote fit metrics representing a single level SRH defect component, a bulk component and a surface component: $1 / \tau_{\mathrm{SRH}}$ (purple), $1 / \tau_{\text {bulk }}$ (green) and $1 / \tau_{\text {surf_init/DA }}$ (orange) respectively. (For interpretation of the references to color in this figure legend, the reader is referred to the web version of this article).

\subsection{Importance of the emitter}

In the previous section, we found that the presence of a diffused emitter can instigate LeTID in n-type silicon. It is unclear, however, whether such affect may be imposed due to thermal processing from the boron diffusion, or perhaps the involvement of the boron dopant itself is also essential. It is also unclear whether boron is a component of the recombination active defect or simply a catalyst. To investigate this, we took a series of unfired boron-diffused and $\mathrm{SiN}_{\mathrm{x}}: \mathrm{H}$ passivated n-type wafers from the same stock as described in Group 1 above. Again, these, wafers were given one of 3 different conditions; which we denote as groups A, $\mathrm{B}$ and C. Group A wafers underwent high-temperature firing at a temperature of $746 \pm 3{ }^{\circ} \mathrm{C}$. 
Following this, wafers were submerged in dilute HF $(2 \% \mathrm{v} / \mathrm{v})$ until hydrophobic to remove the SiNx:H layer. A $2.0 \pm 0.5 \mu \mathrm{m}$ alkaline retexturing process was used to remove the emitters off both surfaces. These wafers were then re-passivated with PECVD SiN $: H$ film on both sides. In group B, wafers are simply fired, followed by the removal and subsequent redeposition of $\mathrm{SiN}_{\mathrm{x}}: \mathrm{H}$ dielectric layers. Finally, wafers in group $\mathrm{C}$ did not undergo an initial firing step. Instead, both the silicon nitride dielectric and emitters were first removed followed by a re-deposition of the passivating layer. Wafers were then fired thereafter, in the absence of a boron emitter. A complete process flow is depicted in Fig. 4. We note that a recent publication by Bredemeier et al. highlighted the relationship between substrate thickness and LeTID maximum defect density, however, a removal of approximately $4 \mu \mathrm{m}$ of silicon from a substrate of $180 \mu \mathrm{m}$ in this work, would only marginally affect the maximum NDD [5].

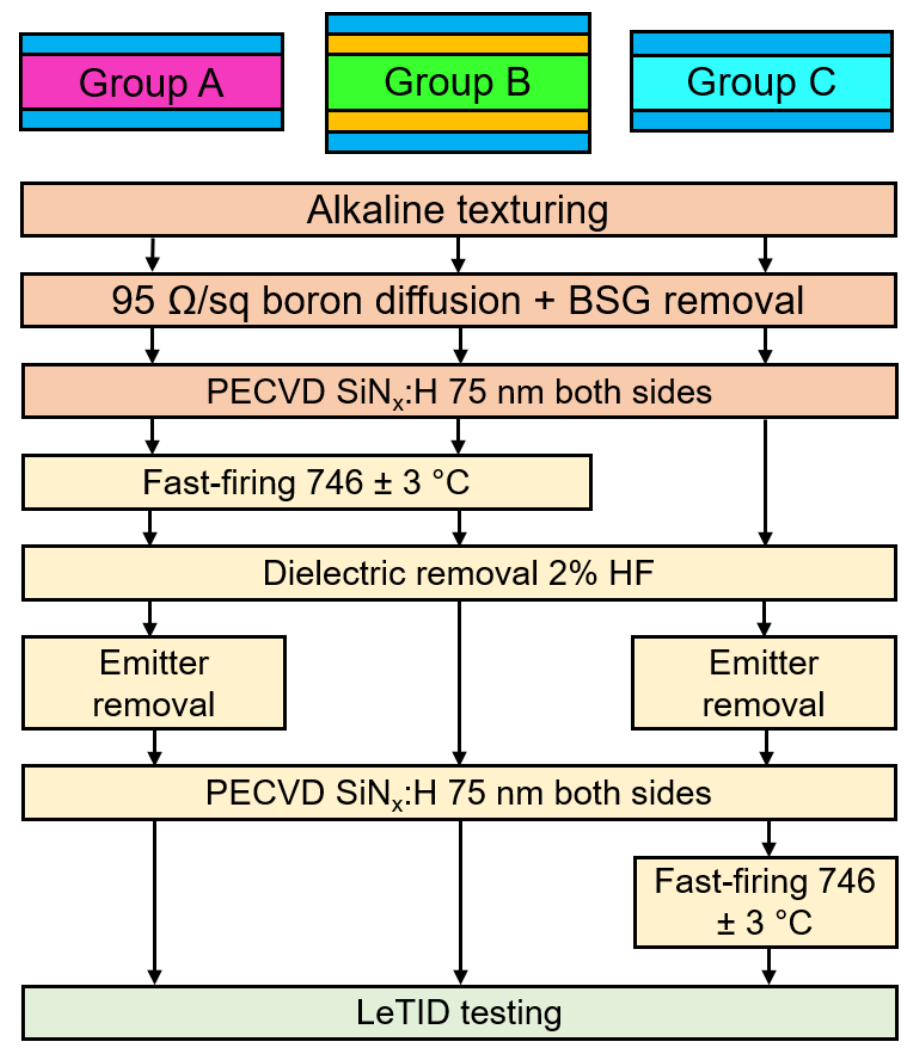

Fig. 4 Process flow diagram investigating the impact of boron-diffused emitters on the generation of LeTID. The final structure before LeTID testing is denoted at the top, with boron diffused $\mathrm{p}^{+}$-emitters shown in yellow and silicon nitride passivation layers in blue. Groups have been coloured for easier reference to results in Fig. 5. (For interpretation of the references to colour in this figure legend, the reader is referred to the web version of this article).

The effect of dark annealing on these various groups was monitored using an in-situ QSSPC measurement and highlighted in Fig. 5. Samples in group A and B, both of which were fired in the presence of a boron emitter manifested in a degradation and recovery of the effective lifetime $\tau_{\text {eff }}$ attributed to a change within the bulk. Given that both groups show very similar defect concentrations and group A no longer has emitters present, it may be suggested that the presence of the emitter and in particular, boron, is not required for the LeTID related lifetime degradation and recovery. Group A appears to recover at an accelerated rate, taking only $10 \mathrm{~h}$ to reach its starting lifetime, however group B requires $50 \mathrm{~h}$. The shift in time 
scales may indicate a change in the precursor concentration, however, we also find that after $10 \mathrm{~h}$, the $\mathrm{J}_{0 \mathrm{~s}}$ of the sample begins to increase (see Fig. 5(b)), which may counteract the recovery process and reduce the recovery rate. The sample with emitters removed prior to silicon nitride re-deposition and firing (Group C), showed an even greater drop in $\tau_{\text {eff. }}$ However, this was found to be a consequence of an increasing $\mathrm{J}_{0 \mathrm{~s}}$ component by up to $30 \%$ from the initial value. The behaviour of this group resembled the non-diffused n-type sample, which also exhibited an apparent surface degradation. It is however, important to determine whether the apparent change in NDD can be explained wholly by the surface. For these samples, a Kane-Swanson linear parametrization of the $\mathrm{J}_{0 \mathrm{~s}}$ was insufficient in providing a good fit, as the injection-dependent lifetime measurements exhibited a slight bow similar in nature to what was observed in [31]. A suitable fit could only be obtained through the addition of an unknown single-level defect situated at $0.4 \mathrm{eV}$ above midgap with an electronto-hole capture cross-section ratio of approximately $k_{\mathrm{SRH}}=0.032$. On non-diffused fired samples, the emitter minority carrier lifetime $\left(\tau_{\text {emitter }}\right)$ was observed to change from $\tau_{\text {emitter, } 0 \mathrm{~h}}=$ $343 \mu$ s to $\tau_{\text {emitter,43h }}=263 \mu$ s. This corresponds to an approximate NDD of $8.8 \times 10^{-4} / \mu$ s, thus correlating closely with the apparent NDD extracted after 43 hours of LeTID testing. Although the extracted bulk lifetime did not vary, it is possible that our observed increase in $\mathrm{J}_{0 \mathrm{~s}}$ may have been due to the formation of a near-surface bulk defect manifesting as an artificial decline in surface passivation quality. In both Group $\mathrm{C}$ and the non-diffused fired samples, this effect appears to be permanent with increasing annealing duration and no subsequent recovery mechanism observed within our examined time frame. These outcomes also convey that the thermal treatment brought about by boron diffusion, is not a contributing driver of LeTID.

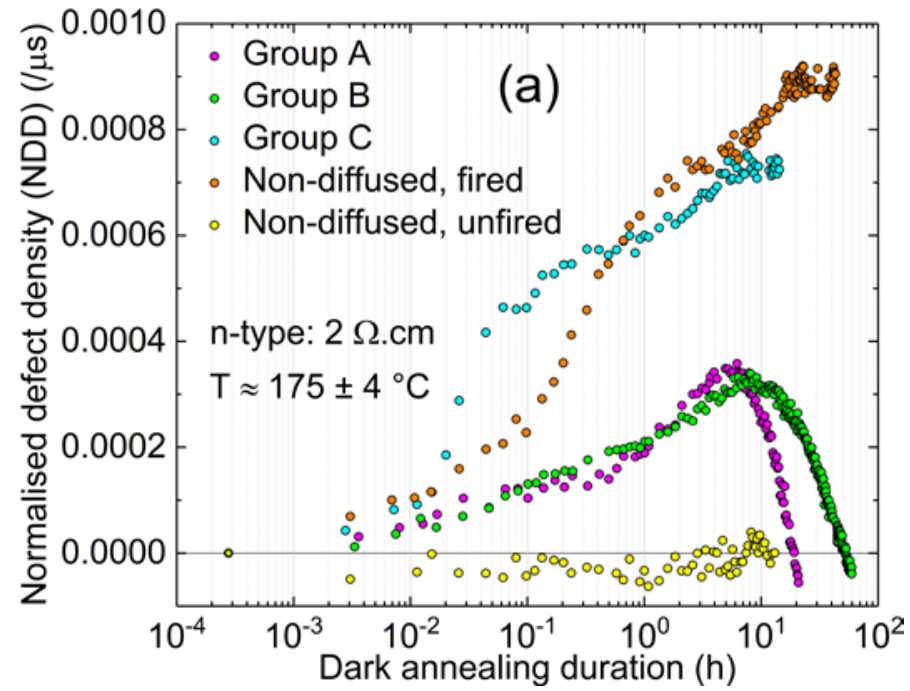




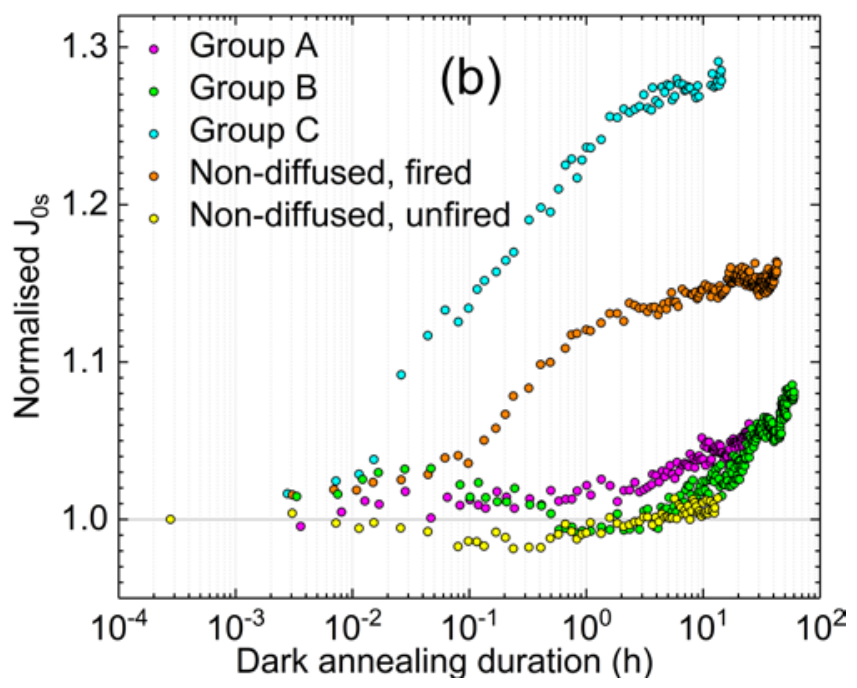

Fig. 5 (a) Normalised defect density as a function of dark annealing duration $175 \pm 4{ }^{\circ} \mathrm{C}$ as measured on lifetime structures in groups A-C prepared on $2 \Omega$.cm n-type silicon wafers. An extra non-diffused, fired (orange) and non-diffused, unfired (yellow) sample from Group 3 above was used as a comparison to the behaviour exhibited by Group C (cyan). Symbols denote in-situ QSSPC lifetime measurements extracted at an injection level of $\Delta n=1.0 \times 10^{15} / \mathrm{cm}^{3}$ with the NDD normalised with respect to the first lifetime measured. (b) Corresponding normalized surface dark saturation current $\left(\mathrm{J}_{0 \mathrm{~s}}\right)$ extracted at an injection level of $\Delta n=1.0 \times 10^{16} / \mathrm{cm}^{3}$. (For interpretation of the references to colour in this figure legend, the reader is referred to the web version of this article).

We also observed that the $\tau_{\text {eff }}$ of unfired, non-diffused samples remained stable (with slight improvements) throughout dark annealing, highlighting that the $\mathrm{J}_{0 \mathrm{~s}}$ increase also only occurs on samples that are fired. In summary, the firing step must be conducted in the presence of an emitter to induce a subsequent bulk degradation during annealing, although the emitter is no longer required during testing in order to observe LeTID-like behaviour. This implies that the diffused region may act as a catalyst to a precursor for LeTID however is no longer necessary for the subsequent degradation and recovery processes. In the following section, we will discuss the possible impact of diffused emitters and dopants with relation to the involvement of hydrogen.

\section{Discussion}

\subsection{Hydrogen induced defects in n-type silicon}

While the exact form of the defects responsible for LeTID remains the subject of debate, there is an increasing body of evidence that is the extent of degradation proportional to the amount of hydrogen present in the silicon [12]. If we accept that the bulk LeTID observed in this paper is caused by the same defect/process responsible for LeTID in p-type silicon (whether multi-crystalline silicon, Cz-Si or FZ-Si), it is reasonable to expect that the extent of this degradation would also be proportional to the bulk hydrogen concentration. Using this assumption there are two main experimental results that we will attempt to explain in this section:

1) Bulk degradation consistent with the behavior of LeTID is observed in n-type samples with a boron emitter present during firing, but is not observed in samples with no diffusion present during firing. 
2) Samples fired with no emitter present exhibit a significant increase in near-surface recombination, which is greatly reduced in those samples that were either not fired with an emitter in place, or not fired at all.

The first point suggests that the bulk hydrogen concentration is higher for samples fired with a boron emitter present than for samples with no emitter in place. This is somewhat surprising given that a boron emitter is expected to be an effective barrier for hydrogen diffusion [32]. There are two closely related possible explanations for why the bulk hydrogen concentration should be lower with no emitter present. One is that the rate of out-diffusion of hydrogen from the samples is much greater with no emitter present, and that during the firing process, most hydrogen is able to leave the wafer, with no significant build up in the bulk material. If we assume that the effects of hydrogen charge states to be negligible at metallization fastfiring temperatures, then the emitter should only act as a barrier for hydrogen effusion during cooling. The other is that hydrogen entering an undiffused n-type wafer remains largely trapped at near surface sites. Both these effects may well be occurring in our samples.

Both of these explanations would require that the solubility of hydrogen in n-type wafers to be much lower than that in p-type. Such evidence for the low solubility of hydrogen in n-type is found in the results of Rizk et al. and Mathiot et al., both showing an accumulation of hydrogen in the near-surface regions $(<2 \mu \mathrm{m}$ deep) of lightly-doped n-type silicon material $[33,34]$. This is further evidenced the observation by Johnson et al. that for similar dopant concentrations of n-type and p-type species, a higher density of precipitated hydrogen will nucleate in n-type material than in p-type material [35]. In both cases, in non-diffused n-type silicon substrates fired in the presence of a $\mathrm{SiN}_{\mathrm{x}}: \mathrm{H}$ dielectric, much of the hydrogen introduced may be situated in a localized subsurface region, leading to a build-up of less mobile hydrogen species. It has been noted by several authors that a significant agglomeration of negatively-charged hydrogen in localized pockets can lead to the formation of hydrogen-induced defects, namely hydrogen platelets and hydrogen-decorated extended defects, albeit at lower temperatures [36-38]. In thermal equilibrium at low-temperatures, due to the Fermi-level determination of the hydrogen charge state, in n-type silicon, the majority of hydrogen within the material would occupy the negative charge $\left(\mathrm{H}^{-}\right)$. Nickel et al. concluded that hydrogen induced platelets would more readily form in n-type silicon due the higher concentration of hydrogen diffusing in the negative charge state [36]. The formation of such defect layers at the undiffused c-Si/SiN $\mathrm{N}_{\mathrm{x}}$ interface has been previously observed using high-resolution transmission electron microscopy measurements and in agreement with our experimental results; has been associated with an increase in the effective surface recombination velocity $\left(S_{\text {eff }}\right)$ and a reduction in surface passivation quality [39-41]. This build-up has also been noted as a possible cause of the surface degradation observed by Keller et al. and Sperber et al. [10,42].

In n-type samples fired with no emitter in place, the increase in near-surface recombination with time could then plausibly be explained either as the growth of existing hydrogen-related defects or the formation of new ones as more hydrogen migrates to that region or dissociates from non-recombinative forms. This could result from additional hydrogen being released from the dielectric layer, but is equally likely a consequence of hydrogen being redistributed from the wafer bulk back to the surfaces (where these sources of this recombination act as 
effective sinks for interstitial hydrogen). This would match with the results of Bredemeier et al. [5] if it is the redistribution of hydrogen from the wafer bulk to the surface that causes the "regeneration" of LeTID. In samples with an emitter present this effect would likely be suppressed, as seen in Figure 5. This is due either to the already reduced recombination lifetimes in the emitter or due to hydrogen preferentially pairing with dopant ions. Provided this effect does not result in deactivation of a significant fraction of dopants within the emitter it would be unlikely to have any observable impact on the excess carrier lifetime.

One other result that requires further explanation is the reduced extent of LeTID observed in samples with a phosphorus emitter when compared with those using a boron emitter. It is well established that significant hydrogen can be transported across phosphorus emitters into p-type substrates, and simulations have predicted that this should be easier than transporting hydrogen across boron emitters with similar doping profiles [43]. However, it should be noted that in this work, the phosphorus emitters had a sheet resistance of $\sim 60 \Omega /$ sq. compared to the $95 \Omega / \mathrm{sq}$. boron emitters. It is therefore likely that the much higher peak doping concentration in the phosphorus emitter blocked significant hydrogen from reaching the ntype bulk.

There are several unanswered questions with this interpretation of our results and alternative explanations. The most glaring question that we cannot answer at present, is why the same agglomeration of hydrogen that we postulate forming under the $\mathrm{SiN}_{\mathrm{X}}: \mathrm{H}$ interface, does not form deeper in the wafer when a boron emitter is present. If this did occur, it would act in the same method to reduce the amount of hydrogen present in the bulk, and hence presumably suppress bulk degradation. The best explanation is that a sufficiently high concentration of hydrogen is required to nucleate the growth of these defects, which is not present after hydrogen has been transported across a boron emitter [43]. Alternately we cannot exclude the possibility that the deposition or firing of the silicon nitride layer may have also led to a surface damage region forming as the results of the thermal process [40]. This could either be required for the nucleation of the hydrogen-related defects or be a different cause of near surface recombination. In addition, our results do not exclude the theory that the bulk recovery mechanism is the result of a stable, recombination-inactive hydrogen state throughout the wafer, such as the $\mathrm{H}_{2 \mathrm{C}}$ dimer identified by Voronkov et al. [44].

\subsection{Implications for the root cause of LeTID: Metallic Impurities}

Although the work presented in this paper does not directly explore the species responsible for LeTID, the manifestation of the defect in n-type silicon provides clues for the validity of candidates previously thought to be responsible for the recombination. In various works, transition metals, metallic point defects and precipitates have been hypothesized to be a component of the root cause for LID. Temperature- and injection- dependent lifetime spectroscopy measurements have suggested the involvement of the titanium interstitial double donor $\left(\mathrm{Ti}_{\mathrm{i}}{ }^{+/++}\right)$, the molybdenum interstitial donor $\left(\mathrm{Mo}_{\mathrm{i}}{ }^{0 /+}\right)$ and the tungsten substitutional donor $\left(\mathrm{W}_{\mathrm{s}}{ }^{0 /+}\right)[30,45]$. Due to the significantly larger capture cross-section for electrons however, the effect of such metallic point defects and other transition metals on low-injection lifetime in n-type silicon would be negligible [46] and should not be responsible for the statistics we report in Fig. 3. Other candidates such as interstitial copper 
and precipitates which has been shown to cause Cu-LID in p-type have also been thought to contribute to LeTID $[47,48]$. However, studies have shown that the nucleation barrier of copper-related defects in n-type is significantly lower due to the position of the Fermi-level close to electron neutrality, such that any minority carrier lifetime degradation would occur directly after $\mathrm{Cu}$ in-diffusion without the need of illumination or excess carrier injection [49,50]. Various other common point defects may also be excluded [19]. Although other uncommon point defects such as $\mathrm{Cr}$ and $\mathrm{Au}$ can have capture cross-section ratios that are orders of magnitude higher for holes than electrons, these slower diffusers are not known to redistribute at the low temperatures used for LeTID testing [51].

Furthermore, despite our hypothesis in [15] previously agreeing with the results of Zuschlag et al., our new findings contradict the observations in p-type silicon that gettering can assist to reduce the appearance of LeTID [52]. We find that by using boron or phosphorus emitter diffusions on n-type silicon; which are known to be excellent gettering processes [53], we instead instigate the defect formation. Further work will be conducted to investigate whether the same phenomenon will occur on p-type with boron-diffused emitters. Although we do not exclude the possibility that hydrogen-metal interactions may result in deep-level defects with recombination activity in n-type silicon [54,55], we believe that it is unlikely for metallic impurities alone to be a cause for LeTID.

Alternatively, our results in Section. 3.2 raises the possibility that the defect species may have multiple energy levels and occupy both acceptor and donor states, such as a negative-U type defect. Some possible examples of such defects include interstitial hydrogen, which obeys negative- $U$ properties centered around the neutral charge state $\left(\mathrm{H}^{0}\right)$ as well as vacancies, which have been suggested to have acceptor and donor vacancy $\left(\mathrm{V}^{-}, \mathrm{V}^{+}\right)$and divacancy $\left(\mathrm{V}^{2-}\right.$ , $\left.\mathrm{V}^{2+}\right)$ states in-addition to an additional neutral charge state $\left(\mathrm{V}^{0}\right)$ [49]. The behavior of vacancies and self-interstitials introduced by dopant migration and incorporation during boron and phosphorus diffusions is well documented and may also be used to explain the observed behaviors between diffused and non-diffused samples [56]. Not only can hydrogen form deep-level recombination active complexes with vacancies [57-59], work by Sopori et al. show an increase in hydrogen solubility in the presence of vacancies and enhanced bulk transport of interstitial hydrogen into bulk silicon [60]. Fahey et al., in contrast, finds that phosphorus diffusion leads to a supersaturation of self-interstitials and an under-saturation of vacancies, which may lead to vacancy-interstitial annihilation and net decrease in nearsurface vacancy concentrations [56]. Although studies of these hydrogen and vacancy complexes with other elements and even with each other as vacancy-hydrogen complexes (V$\mathrm{H}_{\mathrm{x}}$ ) are well known, there is little work identifying the recombination activity of the charged species alone. Whether or not interstitial hydrogen alone may induce recombination activity remains up for debate and further research and theoretical understandings are required.

\section{Conclusion}

In this work, we have demonstrated that, in contrary to previous works, n-type silicon is also susceptible to what is known in p-type studies as LeTID. In the first section, we demonstrated the use of emitter diffusion and firing to instigate carrier induced degradation in n-type 
Czochralski wafers. We further demonstrate that the diffused emitter is essential for the formation of defect precursors. Although hydrogen is important for the passivation of lifetime killing defects in silicon, we believe that hydrogen plays a significant role

\section{Acknowledgements}

Work at UNSW has been supported by the Australian Government through the Australian Renewable Energy Agency (ARENA) (ARENA 1-A060 and 1-SRI001) and the Australian Centre for Advanced Photovoltaics (ACAP), and the Australian Research Council (DE170100620). The views expressed herein are not necessarily the views of the Australian Government, and the Australian Government does not accept responsibility for any information or advice contained herein. Work at Oxford University has been supported by the U.K. Government through the International and Industrial Engagement Fund and the Supersilicon project (EP/ M024911/1), supported by the Engineering and Physical Sciences Research Council (EPSRC). Daniel Chen would like to acknowledge the support and contribution of the Australian Commonwealth Government through the Australian Government Research Training Program Scholarship. The authors would also like to acknowledge Duc Huy Dao, Ly Mai and Qilin Ye for assistance in sample fabrication and Carlos Vargas and Kyung Kim for useful discussions. The authors would also like to thank the commercial partners of the ARENA 1-A060 advanced hydrogenation project and the UK Institution of Engineering and Technology (IET) for their funding support for this work through the A.F. Harvey Engineering Prize.

\section{Conflict of Interest}

The authors declare no conflict of interest.

\section{References}

[1] K. Ramspeck, S. Zimmermann, H. Nagel, A. Metz, Y. Gassenbauer, B. Brikmann, A. Seidl, Light induced degradation of Rear Passivated mc- Si solar cells, in: Proc. 27th Eur. Photovolt. Sol. Energy Conf., 2012: pp. 861-865. doi:10.4229/27THEUPVSEC2012-2DO.3.4.

[2] D. Bredemeier, D. Walter, S. Herlufsen, J. Schmidt, Lifetime degradation and regeneration in multicrystalline silicon under illumination at elevated temperature, AIP Adv. 6 (2016). doi:10.1063/1.4944839.

[3] C.E. Chan, D.N.R. Payne, B.J. Hallam, M.D. Abbott, T.H. Fung, A.M. Wenham, B.S. Tjahjono, S.R. Wenham, Rapid Stabilization of High-Performance Multicrystalline Ptype Silicon PERC Cells, IEEE J. Photovoltaics. 6 (2016) 1473-1479. doi:10.1109/JPHOTOV.2016.2606704.

[4] W. Kwapil, T. Niewelt, M.C. Schubert, Kinetics of carrier-induced degradation at elevated temperature in multicrystalline silicon solar cells, Sol. Energy Mater. Sol. Cells. 173 (2017) 80-84. doi:10.1016/j.solmat.2017.05.066.

[5] D. Bredemeier, D.C. Walter, J. Schmidt, Possible Candidates for Impurities in mc-Si Wafers Responsible for Light-Induced Lifetime Degradation and Regeneration, Sol. 
RRL. 2 (2018) 1700159. doi:10.1002/solr.201700159.

[6] D. Bredemeier, D. Walter, J. Schmidt, Light-induced lifetime degradation in highperformance multicrystalline silicon: Detailed kinetics of the defect activation, Sol. Energy Mater. Sol. Cells. 173 (2017) 2-5. doi:10.1016/j.solmat.2017.08.007.

[7] M.A. Jensen, A.E. Morishige, J. Hofstetter, D.B. Needleman, T. Buonassisi, Evolution of LeTID Defects in p-Type Multicrystalline Silicon During Degradation and Regeneration, IEEE J. Photovoltaics. (2017) 1-8. doi:10.1109/JPHOTOV.2017.2695496.

[8] K. Nakayashiki, J. Hofstetter, A.E. Morishige, T.T.A. Li, D.B. Needleman, M.A. Jensen, T. Buonassisi, Engineering Solutions and Root-Cause Analysis for LightInduced Degradation in p-Type Multicrystalline Silicon PERC Modules, IEEE J. Photovoltaics. 6 (2016) 860-868. doi:10.1109/JPHOTOV.2016.2556981.

[9] C. Chan, T.H. Fung, M. Abbott, D. Payne, A. Wenham, B. Hallam, R. Chen, S. Wenham, Modulation of Carrier-Induced Defect Kinetics in Multi-Crystalline Silicon PERC Cells Through Dark Annealing, Sol. RRL. 1 (2017) 1600028. doi:10.1002/solr.201600028.

[10] P. Keller, Untersuchung und Wasserstoffpassivierung von Defekten in Foliensilizium und multikristallinen Silizium-Materialien, Thesis. (2017).

[11] F. Kersten, J. Heitmann, J.W. Müller, Influence of Al2O3 and SiNx Passivation Layers on LeTID, Energy Procedia. 92 (2016) 828-832. doi:10.1016/j.egypro.2016.07.079.

[12] C. Vargas, K. Kim, G. Coletti, D. Payne, C. Chan, S. Wenham, Z. Hameiri, CarrierInduced Degradation in Multicrystalline Silicon: Dependence on the Silicon Nitride Passivation Layer and Hydrogen Released During Firing, IEEE J. Photovoltaics. 8 (2018) 1-8. doi:10.1109/JPHOTOV.2017.2783851.

[13] S. Wilking, S. Ebert, A. Herguth, G. Hahn, Influence of hydrogen effusion from hydrogenated silicon nitride layers on the regeneration of boron-oxygen related defects in crystalline silicon, J. Appl. Phys. 114 (2013) 194512. doi:10.1063/1.4833243.

[14] F. Fertig, R. Lantzsch, A. Mohr, M. Schaper, M. Bartzsch, D. Wissen, F. Kersten, A. Mette, S. Peters, A. Eidner, J. Cieslak, K. Duncker, M. Junghänel, E. Jarzembowski, M. Kauert, B. Faulwetter-Quandt, D. Meißner, B. Reiche, S. Geißler, S. Hörnlein, C. Klenke, L. Niebergall, A. Schönmann, A. Weihrauch, F. Stenzel, A. Hofmann, T. Rudolph, A. Schwabedissen, M. Gundermann, M. Fischer, J.W. Müller, D.J.W. Jeong, Mass production of p-type $\mathrm{Cz}$ silicon solar cells approaching average stable conversion efficiencies of $22 \%$, in: Energy Procedia, 2017: pp. 338-345. doi:10.1016/j.egypro.2017.09.308.

[15] D. Chen, M. Kim, B. V. Stefani, B.J. Hallam, M.D. Abbott, C.E. Chan, R. Chen, D.N.R. Payne, N. Nampalli, A. Ciesla, T.H. Fung, K. Kim, S.R. Wenham, Evidence of an identical firing-activated carrier-induced defect in monocrystalline and multicrystalline silicon, Sol. Energy Mater. Sol. Cells. 172 (2017) 293-300. doi:10.1016/j.solmat.2017.08.003.

[16] T. Niewelt, M. Selinger, N.E. Grant, W. Kwapil, J.D. Murphy, M.C. Schubert, Lightinduced activation and deactivation of bulk defects in boron-doped float-zone silicon, J. Appl. Phys. 121 (2017) 185702. doi:10.1063/1.4983024. 
[17] T. Niewelt, F. Schindler, W. Kwapil, R. Eberle, J. Schön, M.C. Schubert, Understanding the light-induced degradation at elevated temperatures: Similarities between multicrystalline and floatzone p-type silicon, Prog. Photovoltaics Res. Appl. (2017) 1-10. doi:10.1002/pip.2954.

[18] D. Sperber, A. Heilemann, A. Herguth, G. Hahn, Temperature and light induced changes in bulk and passivation quality of boron-doped float-zone silicon coated with SiNx:H, IEEE J. Photovoltaics. (2017) 1-8. doi:10.1109/JPHOTOV.2017.2649601.

[19] B. Singha, C.S. Solanki, N-type solar cells: Advantages, issues, and current scenarios, Mater. Res. Express. 4 (2017) aa6402. doi:10.1088/2053-1591/aa6402.

[20] G. Coletti, P. Manshanden, S. Bernardini, P.C.P. Bronsveld, A. Gutjahr, Z. Hu, G. Li, Solar Energy Materials \& Solar Cells Removing the effect of striations in n-type silicon solar cells, 130 (2014) 647-651. doi:10.1016/j.solmat.2014.06.016.

[21] C. Renevier, E. Fourmond, M. Forster, S. Parola, M. Le Coz, E. Picard, Lifetime Degradation on n-type Wafers with Boron-diffused and SiO2/SiN-passivated Surface, Energy Procedia. 55 (2014) 280-286. doi:10.1016/j.egypro.2014.08.082.

[22] P.J. Cousins, J.E. Cotter, The influence of diffusion-induced dislocations on high efficiency silicon solar cells, IEEE Trans. Electron Devices. 53 (2006) 457-464. doi:10.1109/TED.2005.863535.

[23] Z. Hameiri, N. Borojevic, L. Mai, N. Nandakumar, K. Kim, S. Winderbaum, Should the refractive index at $633 \mathrm{~nm}$ be used to characterize silicon nitride films?, in: 2016 IEEE 43rd Photovolt. Spec. Conf., IEEE, 2016: pp. 2900-2904. doi:10.1109/PVSC.2016.7750187.

[24] Z. Hameiri, N. Borojevic, L. Mai, N. Nandakumar, K. Kim, S. Winderbaum, LowAbsorbing and Thermally Stable Industrial Silicon Nitride Films With Very Low Surface Recombination, IEEE J. Photovoltaics. 7 (2017) 996-1003. doi:10.1109/JPHOTOV.2017.2706424.

[25] R. Sinton, A. Cuevas, A quasi-steady-state open-circuit voltage method for solar cell characterization, in: 16th Eur. Photovolt. Sol. ..., 2000: pp. 1-4. doi:citeulike-articleid:6901946.

[26] H. Nagel, C. Berge, A.G. Aberle, H. Nagel, C. Berge, A.G. Aberle, Generalized analysis of quasi-steady-state and quasi-transient measurements of carrier lifetimes in semiconductors Generalized analysis of quasi-steady-state and quasi-transient measurements of carrier lifetimes in semiconductors, J. Appl. Phys. 86 (1999) 62186221. doi:10.1063/1.371633.

[27] A. Richter, F. Werner, A. Cuevas, J. Schmidt, S.W. Glunz, Improved parameterization of Auger recombination in silicon, Energy Procedia. 27 (2012) 88-94. doi:10.1016/j.egypro.2012.07.034.

[28] S.W. Glunz, S. Rein, W. Warta, J. Knobloch, W. Wettling, Degradation of carrier lifetime in Cz silicon solar cells, Sol. Energy Mater. Sol. Cells. 65 (2001) 219-229. doi:10.1016/S0927-0248(00)00098-2.

[29] J.M. Dorkel, P. Leturcq, Carrier mobilities in silicon semi-empirically related to temperature, doping and injection level, Solid State Electron. 24 (1981) 821-825. doi:10.1016/0038-1101(81)90097-6. 
[30] A.E. Morishige, M.A. Jensen, D.B. Needleman, K. Nakayashiki, J. Hofstetter, T.A. Li, T. Buonassisi, Lifetime Spectroscopy Investigation of Light-Induced Degradation in $\mathrm{p}$ -type Multicrystalline Silicon PERC, IEEE J. Photovoltaics. 6 (2016) 1466-1472. doi:10.1109/JPHOTOV.2016.2606699.

[31] D. Sperber, A. Graf, D. Skorka, A. Herguth, G. Hahn, Degradation of Surface Passivation on Crystalline Silicon and its Impact on Light Induced Degradation Experiments, (2017) 1-9. doi:10.1109/JPHOTOV.2017.2755072.

[32] P. Hamer, B. Hallam, S. Wenham, M. Abbott, Manipulation of hydrogen charge states for passivation of P-type wafers in photovoltaics, IEEE J. Photovoltaics. 4 (2014) 1252-1260. doi:10.1109/JPHOTOV.2014.2339494.

[33] R. Rizk, P. De Mierry, D. Ballutaud, M. Aucouturier, D. Mathiot, Hydrogen Diffusion in N-Type Silicon.Comparison With P-Type Silicon, MRS Proc. 209 (1990) 573. doi:10.1557/PROC-209-573.

[34] D. Mathiot, Modeling of hydrogen diffusion in n-and p-type silicon, Phys. Rev. B. 40 (1989) 5867-5870. http://prb.aps.org/abstract/PRB/v40/i8/p5867_1.

[35] N.M. Johnson, C. Herring, C. Doland, J. Walker, G.B. Anderson, F.A. Ponce, Hydrogen-Induced Platelets in Silicon: Separation of Nucleation and Growth, Mater. Sci. Forum. 83-87 (1992) 33-38. doi:10.4028/www.scientific.net/MSF.83-87.33.

[36] N.H. Nickel, G.B. Anderson, J. Walker, Hydrogen-induced platelets in disordered silicon, Solid State Commun. 99 (1996) 427-431. doi:10.1016/0038-1098(96)00283-9.

[37] M. Nastasi, T. Höchbauer, J.K. Lee, A. Misra, J.P. Hirth, M. Ridgway, T. Lafford, Nucleation and growth of platelets in hydrogen-ion-implanted silicon, Appl. Phys. Lett. 86 (2005) 1-3. doi:10.1063/1.1900309.

[38] H. Nordmark, R. Holmestad, J.C. Walmsley, A. Ulyashin, Transmission electron microscopy study of hydrogen defect formation at extended defects in hydrogen plasma treated multicrystalline silicon, J. Appl. Phys. 105 (2009). doi:10.1063/1.3073893.

[39] S. Steingrube, P.P. Altermatt, D. Zielke, F. Werner, J. Schmidt, R. Brendel, Reduced passivation of silicon surfaces at low injection densities caused by $\mathrm{H}$-induced defects, 25th Eur. Photovolt. Sol. Energy Conf. Exhib. (2010) 1748-1754. doi:10.4229/25thEUPVSEC2010-2CV.2.9.

[40] S. Steingrube, P. Altermatt, D. Steingrube, J. Schmidt, R. Brendel, Interpretation of recombination at c-Si/SiNx interfaces by surface damage, J. Appl. Phys. 108 (2010) 14506. doi:10.1063/1.3437643.

[41] C.G. Van De Walle, P.J.H. Denteneer, Y. Bar-Yam, S.T. Pantelides, Theory of hydrogen diffusion and reactions in crystalline silicon, Phys. Rev. B. 39 (1989) 1079110808. doi:10.1103/PhysRevB.39.10791.

[42] D. Sperber, A. Graf, D. Skorka, A. Herguth, G. Hahn, Degradation of surface passivation and its impact on light-induced degradation experiments, IEEE J. Photovoltaics. 7 (2017) 1627-1634. doi:10.1109/JPHOTOV.2017.2755072.

[43] P. Hamer, B. Hallam, R.S. Bonilla, P.P. Altermatt, P. Wilshaw, S. Wenham, Modelling of hydrogen transport in silicon solar cell structures under equilibrium conditions, J. Appl. Phys. 123 (2018) 43108. doi:10.1063/1.5016854. 
[44] V. V Voronkov, R. Falster, Formation, dissociation, and diffusion of various hydrogen dimers in silicon, Phys. Status Solidi. 254 (2017) 1600779.

doi:10.1002/pssb.201600779.

[45] C. Vargas, Y. Zhu, G. Coletti, C. Chan, D. Payne, M. Jensen, Z. Hameiri, multicrystalline silicon for solar cells Recombination parameters of lifetime-limiting carrier-induced defects in multicrystalline silicon for solar cells, Appl. Phys. Lett. 92106 (2017). doi:10.1063/1.4977906.

[46] D. Macdonald, L.J. Geerligs, Recombination activity of interstitial iron and other transition metal point defects in p- and n-type crystalline silicon, Appl. Phys. Lett. 85 (2004) 4061-4063. doi:10.1063/1.1812833.

[47] A. Inglese, A. Focareta, F. Schindler, J. Schön, J. Lindroos, M.C. Schubert, H. Savin, Light-induced Degradation in Multicrystalline Silicon: The Role of Copper, Energy Procedia. 92 (2016) 808-814. doi:10.1016/j.egypro.2016.07.073.

[48] J. Lindroos, H. Savin, Formation kinetics of copper-related light-induced degradation in crystalline silicon, J. Appl. Phys. 116 (2014). doi:10.1063/1.4904197.

[49] R. Sachdeva, A.A. Istratov, E.R. Weber, Recombination activity of copper in silicon, Appl. Phys. Lett. 79 (2001) 2937-2939. doi:10.1063/1.1415350.

[50] H. Vahlman, A. Haarahiltunen, W. Kwapil, J. Schön, A. Inglese, H. Savin, Modeling of light-induced degradation due to $\mathrm{Cu}$ precipitation in p-type silicon. II. Comparison of simulations and experiments, J. Appl. Phys. 121 (2017) 195704. doi:10.1063/1.4983455.

[51] M.A. Jensen, A.E. Morishige, S. Chakraborty, R. Sharma, H.S. Laine, B. Lai, V. Rose, A. Youssef, E.E. Looney, S. Wieghold, J.R. Poindexter, J. Correa-Baena, T. Felisca, H. Savin, J.B. Li, T. Buonassisi, Solubility and Diffusivity: Important Metrics in the Search for the Root Cause of Light- and Elevated Temperature-Induced Degradation, IEEE J. Photovoltaics. (2018) 1-8. doi:10.1109/JPHOTOV.2018.2791411.

[52] A. Zuschlag, D. Skorka, G. Hahn, Degradation and regeneration analysis in mc-Si, Conf. Rec. IEEE Photovolt. Spec. Conf. (2016) 1051-1054. doi:10.1109/PVSC.2016.7749772.

[53] S.P. Phang, D. Macdonald, Boron, phosphorus and aluminum gettering of iron in crystalline silicon: Experiments and modelling, Conf. Rec. IEEE Photovolt. Spec. Conf. (2010) 352-356. doi:10.1109/PVSC.2010.5616886.

[54] W. Jost, J. Weber, Titanium-hydrogen defects in silicon, Phys. Rev. B - Condens. Matter Mater. Phys. 54 (1996) R11038-R11041. doi:10.1103/PhysRevB.54.R11038.

[55] W. Jost, J. Weber, H. Lemke, Hydrogen-induced defects in cobalt-doped n-type silicon, Semicond. Sci. Technol. 11 (1996) 22-26.

[56] P. Fahey, R.W. Dutton, S.M. Hu, Supersaturation of self-interstitials and undersaturation of vacancies during phosphorus diffusion in silicon, Appl. Phys. Lett. 44 (1984) 777-779. doi:10.1063/1.94915.

[57] V. V. Melnikov, A Hydrogen - Vacancy Defect In Single-Crystal Silicon, Russ. Phys. J. 59 (2016) 618-625. doi:10.1007/s11182-016-0814-8.

[58] Y.K. Park, S.K. Estreicher, C.W. Myles, P.A. Fedders, Molecular-dynamics study of 
the vacancy and vacancy-hydrogen interactions in silicon, Phys. Rev. B. 52 (1995) 1718-1723. doi:10.1103/PhysRevB.52.1718.

[59] M.A. Roberson, S.K. Estreicher, Vacancy and vacancy hydrogenated complexes in silicon, Phys. Rev. B. 49 (1994).

[60] B.L. Sopori, J.P. Benner, N. Renewable, A. Rohatgi, P. Sana, S.K. Estreicher, Y.K. Park, M.A. Roberson, Hydrogen in silicon: current understanding of diffusion, (1994) 1615-1620. 